\title{
Magnetic network elements in solar cycle 23
}

\author{
Chunlan Jin and Jingxiu Wang \\ Key Laboratory of Solar Activity, National Astronomical Observatories, \\ Chinese Academy of Sciences, Beijing 100012, China. email: cljin@nao.cas.cn
}

\begin{abstract}
In this report, we present our recent effort to understand the cyclic behavior of network magnetic elements based on the unique database from full-disk observations provided by Michelson Doppler Imager on board the Solar and Heliospheric Observatory in the interval including the entire cycle 23. The following results are unclosed. (1) The quiet regions dominate the solar magnetic flux for about 8 years in solar cycle 23 , and from the solar minimum to maximum they contribute $(0.94-1.44) \times 10^{23} \mathrm{Mx}$ flux to the solar photosphere. In the entire cycle 23 , the magnetic flux of the quiet regions is 1.12 times that of active regions. The occupation ratio of quiet region flux equally characterizes the course of a solar cycle. (2) With the increasing magnetic flux per element, the variations of numbers and total flux of the network elements show three-fold scenario: no-correlation, anti-correlation, and correlation with sunspots, respectively. The anti-correlated elements covering the range of $(3-32) \times 10^{18} \mathrm{Mx}$ occupy $77 \%$ of total element number and $37 \%$ of quiet Sun flux. (3) The time-latitude distribution of anti-correlated magnetic elements is out of phase with that of sunspots, while the correlated elements display the similar butterfly diagram of sunspots but with wider latitude distribution. These results imply that the correlated elements are the debris of decayed sunspots, and the source of anti-correlated elements is modulated by sunspot magnetic field.
\end{abstract}

Keywords. Sun: activity, Sun: dynamo, Sun: photosphere

\section{Introduction}

Since 1960s, the small-scale magnetic fields have been observed (Sheeley 1966, Sheeley 1967, Harvey 1971), which are thought to be ubiquitous on the Sun. They contribute most magnetic flux to the solar photosphere, and their flux emerging rate exceeds that of sunspots by two orders of magnitude (Zirin 1987). However, most small-scale magnetic fields still cannot be resolved, and some of their magnetic flux is still hidden (Trujillo Bueno et al. 2004).

A key question of small-scale magnetic fields is to understand their cyclic behaviour. How do they change during a solar cycle? Do they correlate with sunspots? For the subject, some pioneer studies have been reported, which are made either directly by magnetic measurements or indirectly by proxies of small-scale magnetic flux, e.g., the Gband and Ca II K bright points and X-ray bright points. Key points of view are followed:

(1) No cyclic variations: CaII $\mathrm{K}$ emission in solar quiet regions (White \& Livingston 1981); X-ray bright points(Hara \& Nakakubo 2003); magnetic flux of networks (Labonte \& Howard 1982); flux spectrum and total flux of network elements with flux $\leqslant 2.0 \times$ $10^{19} \mathrm{Mx}$ (Hagenaar et al. 2003); Stokes $\frac{Q}{I}$ profile (Trujillo Bueno et al. 2004).

(2) Anti-correlation of small-scale fields with sunspot cycle: number of network bright points in very quiet regions (Muller \& Roudier 1984, 1994); HeII 10830Ådark points in the higher chromosphere (Harvey 1985); coronal X-ray bright points (Davis et al. 1977; Davis 1983; Golub et al. 1979; Sattarov et al. 2002); weak changes of emergence frequency of ephemeral regions with flux less than $(3-5) \times 10^{19} \mathrm{Mx}$ (Hagenaar et al. 2003). 
(3) Correlation with sunspot cycle: more ephemeral regions appeared during active solar condition (Harvey \& Harvey 1974; Harvey 1989); X-ray bright points (Golub et al. 1979; Sattarov et al. 2002, 2010; McIntosh \& Gurman 2005); the number (or magnetic flux) of network structures (Foukal et al. 1991; Meunier 2003); flux distribution and total flux of network concentrations with flux $\geqslant 3.0 \times 10^{19} \mathrm{Mx}$ (Hagenaar et al. 2003).

Understanding the cyclic behaviour of small-scale magnetic fields is an essential task in solar physics. Fortunately, the Michelson Doppler Imager on aboard Solar and Heliosphere Observatory (SOHO/MDI) provides a unique database - full-disk magnetogram covering the complete solar cycle 23 , so that we can learn the cyclic variations of quiet solar magnetic flux and small-scale magnetic elements. However, due to the poor spatial resolution, temporal resolution and sensitivity, only the network magnetic fields are identified and studied in the report.

\section{Observations and data analysis}

We apply the full-disk magnetogram observed based on SOHO/MDI from 1996 September to 2010 February. In order to reduce the noise level, we only select the 5 minute average magnetogram. For a better understanding and comparing with previous solar minima, we extend the MDI data by adding Kitt Peak full-disk magnetogram from 1996 August back to 1994 January.

We assume that the magnetic fields in the photosphere are radial, and then the observed line-of-sight fields are corrected by $\cos (\alpha)$ function, where $\alpha$ for each pixel is defined by its distance from solar disk center (Hagenaar et al. 2003). We only analyze the pixels with angle $\alpha$ less than $60^{\circ}$ due to fewer and fewer magnetic signals with far away solar disk center, and set the flux density of those pixels with angle $\alpha$ lager than $60^{\circ}$ to zero.

For each corrected full-disk magnetogram, a threshold of $15 \mathrm{Mx} \mathrm{cm}^{-2}$ is set to define the active regions and their surroundings, and then a mask is made for each magnetogram. In the mask, only these islands with area larger than $9 \times 9$ pixels are defined as active regions.

\section{Results}

\subsection{Cyclic variation of magnetic flux in quiet region}

For each magnetogram, we compute the magnetic flux and area for active regions and quiet regions, respectively, and analyze their cyclic behaviour (as shown in Figure 1). It is found that quiet regions contribute $(0.94-1.44) \times 10^{23} \mathrm{Mx}$ magnetic flux to solar photosphere from solar minimum to solar maximum in solar cycle 23rd, but quiet solar area decreases from cyclic minimum to maximum by a factor of 1.2. As a comparison, the magnetic flux of active regions increases several orders of magnitude from solar minimum to maximum. Furthermore, in the entire solar cycle 23rd, the monthly average magnetic flux of quiet regions is 1.12 times that of active regions.

We also compute the ratio of quiet solar magnetic flux to solar total flux, and here we call it flux occupation by quiet Sun, which is shown by the purple symbols ' $\mathrm{X}$ ' in the right panel of Figure 1. It is interestingly noticed that the flux occupation by quiet region also characterizes the course of solar cycle, just like sunspots. However, the maximum flux occupation by quiet region marks the minimum of solar cycle. From 2007 July to 2009 October, the flux occupation by quiet region is larger than $90.0 \%$ for 28 continuous months, which describes the grand minimum between solar cycles 23 and 24 . During such a low active level, there are 25 months for which magnetic flux of active regions is lower 
than $10^{22} \mathrm{Mx}$. Comparing with the solar minimum of cycle 22 , there are only 7 months with flux occupation by quiet region larger than 90\%, i.e., from 1995 December to 1996 April and from 1996 December to 1997 January. In addition, during the complete solar cycle 23, i.e., from 1996 October to 2008 December, the quiet regions dominate the solar magnetic flux for almost 8 years.

\subsection{The cyclic behaviour of network magnetic elements}

After excluding active regions, we apply the magnetic field value of $6 \mathrm{G}$ as a threshold to make a mask for each quiet magnetogam from 1996 September to 2010 February, and identify the magnetic concentration with area more than 10 pixels as network magnetic elements. According to the magnetic flux per element, we divided the identified magnetic elements into 96 sub-groups. For each sub-group, we study its cyclic variation and compute the correlation coefficient with sunspots, which is shown in Figure 2. With the increasing magnetic flux per element, a remarkable three-fold scenario of the variations of numbers and total flux of the network elements is found: no-correlation, anti-correlation, and correlation with sunspots. The anti-correlated magnetic elements, covering the magnetic flux range of $(3-32) \times 10^{18} \mathrm{Mx}$, occupy $77 \%$ of magnetic elements number and $37 \%$ of network magnetic flux.

In order to further explore the cyclic behaviour of network elements, we analyze the flux spectrum of network magnetic elements, which is shown in Figure 3. From solar minimum to maximum, the distribution of magnetic elements with magnetic flux (3$32) \times 10^{18} \mathrm{Mx}$ gradually decreases, which shows anti-correlation variation with sunspots, but the distribution of magnetic elements with flux $(4-38) \times 10^{19} \mathrm{Mx}$ shows in-phase variation with sunspots and reaches the maximum in the year 2000, 2001, and 2002. For the network magnetic elements with flux around $3 \times 10^{19} \mathrm{Mx}$ and less than $3 \times 10^{18} \mathrm{Mx}$, their distribution displays almost no solar cycle variation.

\subsection{The time-latitude distribution of network magnetic elements}

We also analyze the cyclic variation of latitude distribution of the network elements, which is shown in Figure 4. The number of network elements in each latitude belt has been corrected by the ratio of the quiet region area to corresponding latitude belt area. Panel A(1) and A(2) show the latitude distribution of in-phase magnetic elements in solar cycle 23 , where the red ' + ' symbol shows the sunspots butterfly diagram. It is noticed that these correlated magnetic elements show similar butterfly diagram with sunspots but wider latitude distribution. However, for the anti-correlated magnetic elements, the distribution latitudes gradually become narrow towards solar equator from solar minimum to maximum, and then gradually become wider toward higher latitude belt.
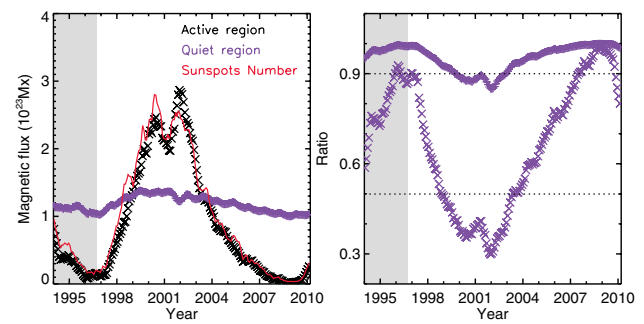

Figure 1. Cyclic variations of magnetic flux of active regions and quiet regions. Left panel: the flux variation of quiet regions (purple line) and active regions (black line). The red line means the variation of sunspots in this period . Right panel: the variation of flux occupation (purple ' $\mathrm{X}$ ' symbols) and area occupation (purple '+' symbols) for quiet regions 


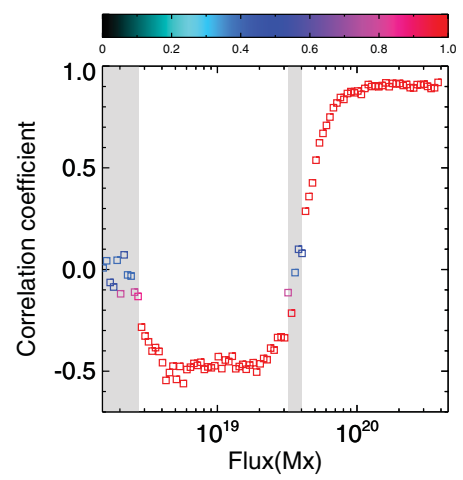

Figure 2. Distribution of correlation coefficients of the sunspot number and the network elements number. The color bar means the confident level of correlation.

\section{Discussion}

The magnetic nature of small-scale magnetic activities is still an unresolved and debatable problem. At the present time, we can confirm that the magnetic elements and smallscale magnetic proxies are not one-to-one corresponded (Zhao et al. 2009). Mehltretter (1974) deduced that the network bright points represent magnetic flux concentration with a mean flux $4.7 \times 10^{17} \mathrm{Mx}$ with an assumption of one-to-one correspondance between network bright points and magnetic elements. Later, Muller \& Roudier (1984, 1994) deduced an average flux of $2.5 \times 10^{17} \mathrm{Mx}$ for network bright points under the same assumption. However, these values of magnetic flux for small-scale magnetic proxies are out of reach by the current database because the minimum magnetic element in the study has magnetic flux with $1.5 \times 10^{18} \mathrm{Mx}$. Using the magnetogram observed from Kitt Peak with a resolution 2.5 arc-second, Golub et al. (1977) found that for a typical Xray bright point, the average magnetic flux is $2 \times 10^{19} \mathrm{Mx}$, which is falling in the flux range of anti-correlated component $\left((3-32) \times 10^{18} \mathrm{Mx}\right)$. These anti-correlated magnetic

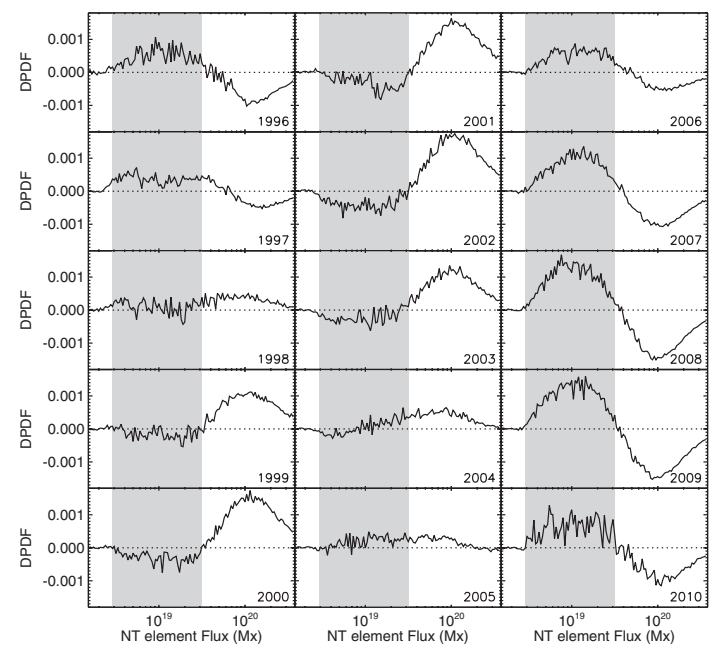

Figure 3. The differential probability distribution function (DPDF), i.e, flux spectrum for network magnetic elements. DPDF is computed by the difference of probability distribution function between yearly network elements and cyclic network elements. 


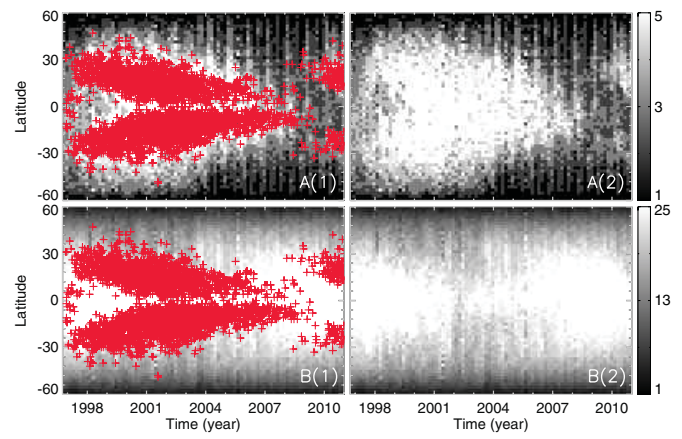

Figure 4. The time-latitude distribution of correlated and anti-correlated magnetic elements

elements identified in the study seem to provide new insight for anti-correlated variation of small-scale magnetic activities with sunspots.

The observations show that the small-scale magnetic fields come from several sources: fragmentation of active region, flux emergence, coalescence of inter-network flux, and products of dynamic interaction among different sources of magnetic flux. The threetype small-scale magnetic elements identified in the study perhaps have an immediate hint for solar magnetic field. For the no-correlated magnetic elements resolved by MDI, they perhaps originate from the local dynamo operating near photosphere. It can not be excluded that the non-correlation of the smallest elements is an effect of poor sensitivity in observing smaller elements. For the correlated magnetic elements, they follow the sunspot butterfly diagram but wider latitude distribution, so they are likely to be debris of decayed sunspots.

The key issue is how to understand the majority magnetic elements that show anticorrelated variation with sunspots in the solar cycle. They are likely to be affected or controlled by the global magnetic field. Here, we consider a few possibilities. First, at solar maximum, the smaller magnetic elements created by the local dynamo have more opportunity to encounter sunspots and their fragment so that speed up the diffusion of active region. Accompanying the diffusion, number of smaller elements is reduced. Second, it is also possible that the sunspot fields at solar maximum suppress the global convection in a sense. As a result, the local dynamo has been abated, and the network magnetic elements created by local dynamo decrease. Another possibility is that the anti-correlated component represents the recycling of parts of previously diffused or submerged magnetic flux from sunspot.

\section{Conclusion}

Based on the SOHO/MDI full-disk magnetogram, the cyclic variation of quiet solar magnetic field is studied. Quiet regions contribute $(0.94-1.44) \times 10^{23} \mathrm{Mx}$ flux from solar minimum to maximum. The area of quiet region decreases 1.2 times from solar minimum to maximum, but total flux increase 1.53 times. The flux occupation by quiet region can also describe the course of the solar cycle, and obviously characterize the grand solar minima of cycle 23-24. Three categories of small-scale elements are found with increasing magnetic flux per elements, and the variations of network element number and network flux successively show no-correlation, anti-correlation, and correlation with sunspots. Anti-correlated component occupies $77 \%$ of total elements and $37 \%$ of flux, which seems to offer an interpretation of anti-correlated variation of small-scale magnetic activities with sunspots. 


\section{Acknowledgements}

This work is supported by the National Basic Research Program of China (2011CB811403) and the National Natural Science Foundations of China (11003024, 40974112, 40890161, 10921303, and 11025315).

\section{References}

Davis, J. M. 1983, Sol. Phys., 88, 337

Davis, J. M., Golub, L., \& Krieger, A. S. 1977, ApJ, 214, L141.

Foukal, P., Harvey, K., \& Hill, F. 1991, ApJ, 383, L89

Golub, L., Krieger, A. S., Harvey, J. W., \& Vaiana, G. S. 1977, Sol. Phys., 53, 111

Golub, L., Davis, J. M., \& Krieger, A. S. 1979, ApJ, 229, L145

Hagenaar, H. J., Schrijver, C. J., \& Title, A. M. 2003, ApJ, 584, 1107

Hara, H. \& Nakakubo, K. 2003, ApJ, 589, 1062

Harvey, J. 1971, Publ. Astron. Soc. Pacific, 83, 539

Harvey, K. 1985, Aust. J. Phys., 38, 875

Harvey, K. 1989, Bull. American Astron. Soc., 21, 839

Harvey, K. \& Harvey, J. 1974, Bull. American Astron. Soc., 6, 288

Labonte, B. J. \& Howard, R. 1982, Sol. Phys., 80, 15

Mehltretter, J. P. 1974, Sol. Phys., 38, 43

Meunier, N. 2003, A\&BA, 405, 1107

McIntosh, S W. \& Gurman, J. B. 2005, Sol. Phys., 228, 285

Muller, R. \& Roudier, T. 1984, Sol. Phys., 94, 33

Muller, R. \& Roudier, T. 1994, Sol. Phys., 152, 131

Sattarov, I., Pevtsov, A. A., Hojaev, A. S., \& Sherdonov, C. T. 2002, ApJ, 564, 1042

Sattarov, I., Pevtsov, A. A., Karachik, N. V., Sherdonov, C. T., \& Tillaboev, A. M. 2010, Sol. Phys., 262, 321

Sheeley, N. R. Jr. 1966, ApJ, 144, 723

Sheeley, N. R. Jr. 1967, Sol. Phys., 1, 171

Trujillo Bueno J., Shchukina, J. N., \& Asensio Ramos, A. 2004, Nature, 430, 326

White, O. R. \& Livingston, W. C. 1981, ApJ, 249, 798

Zirin, H. 1987, Sol. Phys., 110, 101

Zhao, M., Wang, J. X., Jin, C. L., \& Zhou, G. P. 2009, RAA, 9, 933 